\title{
Norois
}

Environnement, aménagement, société

$221 \mid 2011$

Innovations et agricultures urbaines durables

\section{Géographie amoureuse du monde}

\section{Christophe Grenier}

\section{(2) OpenEdition \\ Journals}

Édition électronique

URL : http://journals.openedition.org/norois/3822

DOI : $10.4000 /$ norois.3822

ISBN : 978-2-7535-1838-4

ISSN : 1760-8546

Éditeur

Presses universitaires de Rennes

Édition imprimée

Date de publication : 30 décembre 2011

Pagination : 126-129

ISBN : 978-2-7535-1786-8

ISSN : 0029-182X

Référence électronique

Christophe Grenier, «Géographie amoureuse du monde », Norois [En ligne], 221 | 2011, mis en ligne le 30 décembre 2011, consulté le 03 mai 2019. URL : http://journals.openedition.org/norois/3822 ; DOI : $10.4000 /$ norois.3822

(c) Tous droits réservés 


\section{COMPTES RENDUS BIBLIOGRAPHIQUES}

DuChÊNE François (dir), 2010. Cités ouvrières en devenir. Ethnographies d'anciennes enclaves industrielles, Publications de l'Université de Saint-Étienne, coll. « Dynamiques métropolitaines », 270 p. ISBN 978-2-86272-526-0, $20 €$.

François Duchêne s'entoure d'onze contributeurs d'horizons professionnels divers - du géographe à l'anthropologue, de l'ethnologue à l'ingénieur, de l'architecte à l'urbaniste, du photographe au militant - pour nous livrer un ouvrage stimulant sur ce qu'ils nomment les enclaves industrielles.

Sept analyses-portraits de cités ouvrières de la région Rhône-Alpes amènent le lecteur vers des réflexions bien plus globales sur la ville et comment on y habite, éclairant du passé des réalités contemporaines, parfois complexes. Outre l'intelligence générale du propos, la qualité première de ce travail est de mettre l'habitant au cœur de la réflexion, proposant comme matériau à l’analyse le vécu, la mémoire, la parole de ceux qui vivent dans ces cités ouvrières, depuis presque toujours maintenant.

L'ouvrage est organisé en trois parties qui nous proposent tour à tour, la mémoire, la normalisa-

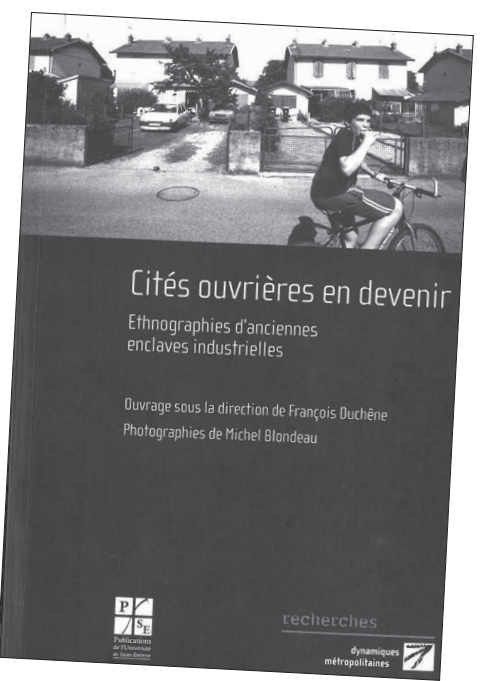
tion et la rencontre/la confrontation au dehors, à l'Autre.

Un retour historique complète l'introduction avec un cheminement et des hypothèses intéressantes sur les conditions d'émergence de cette forme d'habitat. La pensée hygiéniste dès le $\mathrm{XIX}^{\mathrm{e}}$ siècle certes, mais aussi le modèle colonial d'organisation industrielle et agricole sont la toile de fond de ces initiatives du capitalisme dit paternaliste. On croit volontiers les auteurs qui évoquent la probable collusion entre les colons et les grands industriels métropolitains pour penser la domination des hommes et des lieux, même sous couvert d'un catholicisme social.

S'ouvre alors l'inéluctable question de la mémoire ouvrière. Elle fait partie des lieux et elle vieillit avec. C'est une mémoire sensible, tout en ambivalence entre violence et vie ordinaire. La violence est multiple, le patronat dominant impose une morale, un contrôle social puissant et permanent à l'église, à l'usine et à l'école. L'État coopère parfois en acceptant par exemple d'affecter une section des gardes mobiles sur le site de la TASE - Textile Artificiel du Sud-Est - à Vaux-en-Velin à la demande du patron. La mémoire se raconte par les mots recueillis, mais elle se vit aussi dans les corps. Les anciens ouvriers sont vieux, malades ou mutilés par des années de mines ou d'usines. La mémoire est empreinte de violence mais elle est aussi faite de souvenirs d'enfance, d'âge d'or, de solidarités, d'appropriation affective des lieux : la cité et l'usine, l'une avec l'autre. C'est une mémoire tout en contraste, d'haine et d'amour.

Puis le patron et l'usine disparaissent, mais la cité reste. Ces enclaves deviennent des quartiers, du moins tentent de devenir des quartiers normalisés. Les systèmes de gestion changent (les pouvoirs publics remplacent les patrons) au rythme des « réajustements économiques » modifiant inexorablement les manières d'habiter. La 
gratuité devient conflit, la maison devient pavillon et l'appropriation devient stratégie résidentielle.

C'est alors que le microcosme, savamment organisé par la logique industrielle et ardemment défendu par les habitants qui y protègent leur vie, doit s'ouvrir à la ville, à l'Autre. Ces enclaves doivent faire face aux nouvelles logiques métropolitaines qui les cernent. Ce n'est pas simple, forcément. Les cheminots doivent cohabiter avec les « cassociaux », les « anciens » doivent voisiner avec les « nouveaux ». Les réflexes d'hostilités et de repli sont fréquents, sans pour autant être systématiques.

La question de la patrimonialisation, que les auteurs mettent en question dans l'introduction et la conclusion, pourrait être le moyen de répondre au désir de reconnaissance qu'ont ces habitants-travailleurs. C'est un enjeu de classe une fois encore. Le sentiment de tout se faire voler, même son histoire. Gare toutefois au factice et à l'instrumentalisation, préviennent les auteurs.

Il est à regretter cependant que l'apport iconographique de cet ouvrage soit si léger, malgré la présence d'un photographe dans l'équipe. Les photographies sont nombreuses certes, mais les légendes sont en dernières pages, ce qui ne facilitent pas la promenade visuelle du lecteur. Elles sont aussi très vides, ces cités ressemblent à des villes fantômes. Ni femmes, ni hommes, ni enfants. Où sont donc tous ces habitants dont on nous parle?

Le lecteur aurait aussi apprécié des cartes de localisation, mais ce qui manque le plus, ce sont sûrement des schémas de la morphologie urbaine de ces cités. Nous aurions aimé voir, pour mieux comprendre, la forme de ces utopies patronales.

Ces manques sont regrettables mais ne suffisent pas à nuire à la qualité de ce travail qui soulève plusieurs questions essentielles pour tous ceux qui s'intéressent à la ville. Il traite des formes urbaines qui naissent des organisations socio-économiques (donc politiques) et des habitants, avec entre les deux le logement, au cœur de cette double préoccupation. On referme alors ce livre en réfléchissant à l'imposition d'un modèle urbain et à ses évolutions, à la manière dont on passe de la ville planifiée (la cité ouvrière comme «ville nouvelle ») à la ville spontanée. On pense aussi aux habitants, à leurs mémoires et à leurs espoirs, aux rapports sociaux qui se jouent. L'idée de l'entre soi fait à la fois ville et anti-ville et ces exemples décrivant un enfermement social certain, un système ségrégatif puissant et un fort contrôle social sont bien évidemment des éléments intéressants pour penser la ville aujourd'hui.

Fanny Vuaillat

Brunel Sylvie, 2011, Géographie amoureuse du monde, Paris, JC Lattès, 278 p.

Dans sa Géographie amoureuse du monde, Sylvie Brunel survole la géographie et le monde, en neuf courts chapitres entachés d'erreurs, dont celles qui ont trait au Pacifique donnent un aperçu : Bora-Bora est un atoll (p. 77), Cook disparaît en 1777 (p. 82), Vanikoro est aux Fidji (p. 82), Rapa Nui a moins de $40 \mathrm{~km}^{2}$ et compte plus de 300 espèces endémiques (p. 90), « le monde polynésien englobe la Nouvelle-Calédonie, la Micronésie et la Mélanésie » (p. 144)... On relève aussi, à propos du Pacifique, des généralisations surprenantes : ainsi, «la violence du climat et le manque de ressources ont longtemps fait des îles polynésiennes des enfers de la faim » (p. 81), ce qui n'est exact que pour certains atolls, d'ailleurs peuplés en dernier. Ou encore, «l'insularité rend les îliens vulnérables à toutes sortes de croyances, entretenues par 
le huis clos dans lequel mijotent les rancœurs et les peurs » (p. 90) ; S. Brunel aurait du lire l'œuvre de J. Bonnemaison sur Vanuatu.

Pourtant, assure l'auteur, «l'université m’a donné des réflexes académiques : comment trouver et lire tout ce qui a été publié sur une question avant de prétendre apporter sa propre pierre » (p. 176). Or S. Brunel consacre un chapitre à l'île de Pâques en ne s'appuyant que sur deux références. Ou encore, l'auteur fait deux fois allusion (p. 132 et p. 152) à un "numerus clausus » touristique aux Galapagos, qui n'a pourtant jamais existé; elle n'a manifestement pas lu grand chose non plus sur ces îles.

De plus, si S. Brunel mentionnne ses amis, jamais elle ne nomme les « nouveaux flagellants à la jouissance masochiste » (p. 19) auxquels elle s'en prend. On aurait aimé voir cités les travaux de ceux qui sont l'objet des sarcasmes de l'auteur, règle élémentaire de déontologie.

La thèse de S. Brunel est simple : l'action humaine sur la planète est bonne, tandis que la nature est mauvaise. L'humanité est accusée par des «imprécateurs » qui se font ainsi de la publicité, alors qu'en réalité, "nous n'avons jamais aussi bien vécu » (p. 20). Et sa méthode est simplissime: S. Brunel dénigre, caricature et généralise systématiquement. Examinons sa « démonstration».

« La terre qu'ont façonnée les hommes est belle, accueillante, fertile et pleine de diversité » (p. 12). Ces généralités, répétées tout au long du livre, reviennent à nier que, d'une part, des centaines de millions de personnes survivent dans des conditions inhumaines, au sein d'environnements pourtant produits par les sociétés, et d'autre part à ignorer que la rapide érosion de la biodiversité résulte de certaines actions humaines. De plus, et paradoxalement, l'auteur décrit des environnements ravagés par l'homme tels que le «territoire défiguré » par le tourisme de certains lieux en Camargue (p. 54-56), la pollution du Rhône (p. 64) et de certains lagons (p. 107), la destruction des mangroves (p. 102), etc. Pourquoi soutenir alors que « le monde [est] beau partout où l'homme a imprimé sa marque » (p. 12)?

À l'opposé, « la nature, c'est l'impitoyable victoire du fort sur le faible, la sélection naturelle qui élimine tout, les fragiles, les âgés, les malades... Une antithèse exacte de cette merveilleuse invention humaine qu'est la démocratie» (p. 254). Une nature nazie contre une humanité démocratique... On comprend mieux la posture de "résistante » qu'affiche S. Brunel face aux « prédicateurs alarmistes » qui, « s'ils en avaient la possibilité, m’exécuteraient séance tenante en place publique ( (p. 19)! Pour S. Brunel, qui déplore une déprise agricole l'empêchant de se promener à sa guise en raison des taillis (p. 11, p. 257), la seule nature qui vaille doit être « fréquentable, apprivoisée et hospitalière » (p. 259), tandis que « c'est dans les forêts profondes, les hautes montagnes, les étendues arides ou gelées que l'homme perd ses repères et devient vulnérable » (p. 11). Bien des peuples vivent pourtant dans ces contrées, et plusieurs géographes ont étudié leur ingénieuse adaptation à ces environnements, certes hostiles pour les promeneurs du dimanche.

De fait, la hantise de S. Brunel est la conservation de cette nature sauvage qu'elle déteste, car «figer un écosystème le détruit », alors qu'il « n'est jamais immobile et ne cesse d'évoluer, quitte à disparaître et à être remplacé » (p. 62). La conservation de la nature ne vise pas à "figer des écosystèmes », ce qui est absurde et impossible, mais à les préserver de certains usages destructeurs en cours dans le reste du monde. S. Brunel récidive à propos des territoires, en utilisant le procédé conservateur consistant à naturaliser les faits sociaux : "il est vain de vouloir figer un territoire. Comme les écosystèmes, ils ne cessent d'évoluer et c'est la condition de leur pérennité » (p. 134). Les territoires - autrement dit les sociétés, comme les écosystèmes, évoluent constamment, ce qui justifie qu'ont les « désenclave » au profit d'un développement qui est naturel puisque fondé sur la croissance, à l'inverse de leur « mise sous cloche » par des 
conservationnistes assimilés aux colonisateurs (p. 217). CQFD : le développement est progressiste, tandis que la conservation, elle, est conservatrice!

Pour S. Brunel, ce progressisme est illustré par Abou Dhabi, qui « en moins d'une génération, a su passer de la violence de la nature à la domination d'une nature façonnée et recréée, y compris dans sa biodiversité » (p. 169). Abou Dhabi, « oasis de tolérance et de vie » (p. 27), invente « la croissance propre » (p. 167). Bien sûr, «l'eau est dessalée à grands frais, et la population est composée à $80 \%$ de travailleurs immigrés » (p. 168), mais «le pétrole créé de l'intelligence » (p. 155)... «lorsqu'il est bien géré » (p. 170). C'est probablement la première fois qu'un émirat du Golfe est promu comme exemple à suivre en matière de durabilité.

Plus généralement, S. Brunel s'attaque à toute remise en question du productivisme, et en particulier à la notion d'empreinte écologique, qui «ne tolère que le mode de vie d'un paysan bolivien ou burkinabé - pas d'énergie autre que musculaire, pas de mobilité, pas de chauffage ou de climatisation, et bien sûr la nourriture la plus frugale et la moins variée possible» (p. 259). Ces outrances visent à faire croire que les critiques du productivisme n'ont d'autre solution à proposer que le retour en arrière, antienne des technocrates. Ainsi, par exemple, S. Brunel soutient-elle, face aux essais de réduire le trafic automobile en ville, que " personne ne voudrait voir revenir les attelages d'antan et les innombrables accidents de circulation causés par l'embouteillage de voitures à cheval » (p. 123). De même, la « disneylandisation du monde »- ou sa mise en scène touristique - est la solution prônée pour certaines régions, parce que cela «leur évite de disparaître » (p. 125). C'est aberrant : ce ne sont pas les régions qui s'effacent mais, comme le dit l'auteur pour s'en gausser, les «forêts brûlées, les glaciers fondus, l'eau évaporée, l'air et la terre pollués, les animaux éliminés »... (p. 21). Néanmoins, le message est clair : Disneyland ou la disparition...

S. Brunel utilise la caricature en guise d'argument scientifique: «Peur de manquer de ressources, peur du nucléaire, peur des manipulations génétiques, peur du nombre... Calendrier maya, prophétie de Nostradamus, toutes les prédictions les plus alarmistes sont convoquées » (p. 18). Il est irrationnel de mettre en doute le dogme d'une croissance illimitée au prix de techniques porteuses de destructions irréversibles, et les milliers de chercheurs de toutes disciplines qui travaillent sur le réchauffement climatique ou l'érosion de la biodiversité planétaire sont donc des charlatans. Qu'on ne s'y trompe pas, S. Brunel ne défend pas son genre de vie, mais «l'humanité, (qui) est mise en accusation : elle ne saurait que proliférer et détruire, agissant sans conscience » (p. 12). Or ce n'est pas «l'humanité » en tant que telle qui menace son propre habitat terrestre, mais un mode de développement productiviste et le genre de vie qui l'accompagne, historiquement datés.

La liste de ses publications grand public et la consultation d'Internet montrent que S. Brunel bénéficie d'une large couverture médiatique. Pourtant, d'après l'auteur, ce sont « ceux qui se complaisent dans l'imprécation et accablent le monde de prédictions dramatiques qui ont leur couvert mis en permanence à la table des médias » (p. 19). Or l'auteur « ne voit pas pourquoi nous devrions vivre environnés de prophètes de malheur» (p. 12), alors que « la terre est un banquet : jamais nous n'avons aussi bien vécu, autant produit, autant voyagé » (p. 16). S. Brunel imagine que tout le monde veut vivre comme elle, feint de croire que c'est possible, et n'a pas la moindre idée des conséquences catastrophiques de la diffusion de son propre genre de vie - minoritaire en France même - au reste de l'humanité.

L’idéologie de S. Brunel est celle du dépassement des limites biophysiques de la planète : « il n'existe nul obstacle infranchissable, nulle contrainte physique insurmontable dès lors que se déploie l'intelligence des hommes [...], comme le montre Abou Dhabi » (p. 27). L'éloge du productivisme atteint des sommets dans le chapitre consacré aux 
agriculteurs. S. Brunel justifie l'agriculture productiviste et ses pollutions par la lutte contre les famines : les algues vertes «ne datent pas d'aujourd'hui » et la « haute toxicité » des pesticides est normale, car « on n'élimine pas les ravageurs avec de l'eau de rose » (p. 250). De plus, la progression des terres cultivées dans le monde n'est pas une menace pour la biodiversité, puisque « une prairie capte plus de $\mathrm{CO}^{2}$ qu'une forêt et offre en termes de biodiversité une variété de plantes et d'animaux incroyablement nombreux » (p. 244). On se demande bien pourquoi certains se préoccupent alors de la déforestation en Amazonie.

«Être géographe, c'est lire le monde » (p. 13), nous dit l'auteur à juste titre. S. Brunel ne lit pas le monde, mais râbache le credo de ceux qui détruisent la beauté de la Terre.

Christophe Grenier Université de Nantes, LETG-Géolittomer 